\title{
Hydrodynamic modes, Green-Kubo relations, and velocity correlations in dilute granular gases
}

\author{
J Javier Brey, M J Ruiz-Montero, P Maynar and M I García de Soria
}

Física Teórica, Universidad de Sevilla, Apartado de Correos 1065, E-41080, Sevilla, Spain

E-mail: brey@us.es and majose@us.es

Received 27 October 2004, in final form 13 December 2004

Published 3 June 2005

Online at stacks.iop.org/JPhysCM/17/S2489

\begin{abstract}
It is shown that the hydrodynamic modes of a dilute granular gas of inelastic hard spheres can be identified, and calculated in the long wavelength limit. Assuming they dominate at long times, formal expressions for the NavierStokes transport coefficients are derived. They can be expressed in a form that generalizes the Green-Kubo relations for molecular systems, and it is shown that they can also be evaluated by means of $N$-particle simulation methods. The form of the hydrodynamic modes to zeroth order in the gradients is used to detect the presence of inherent velocity correlations in the homogeneous cooling state, even in the low density limit. They manifest themselves in the fluctuations of the total energy of the system. The theoretical predictions are shown to be in agreement with molecular dynamics simulations. Relevant related questions deserving further attention are pointed out.
\end{abstract}

\section{Introduction}

Rapid granular flows exhibit a phenomenological behaviour that is similar in many ways to that of ordinary fluids. So it is not surprising that the methods of statistical mechanics and kinetic theory are being successfully extended to describe them. The primary feature of grains that must be captured in any idealized model is the inelasticity of collisions. The prototype is a system of smooth inelastic hard spheres (or discs in two dimensions) whose energy loss in collisions is characterized by a coefficient of normal restitution. In real granular media, this coefficient depends on the relative velocity of the colliding particles [1], but for the sake of simplicity, and expecting to retain the main qualitative features, it will be considered here as a material constant.

At the most fundamental level of particle dynamics, the effect of the inelasticity is easily incorporated by changing the binary collision rule. As in the elastic case, the dynamics of the system consists of a sequence of free streaming between collisions and instantaneous velocity changes on binary collisions. Therefore, a statistical mechanics description can be formulated 
by means of the Liouville equation of the system [2,3]. From a conceptual point of view, this description has the same degree of justification as that of inherently non-equilibrium molecular systems. Proceeding to a more mesoscopic description, as provided by kinetic theory, is a more difficult and controversial problem. Formally, the same methods as developed for hard sphere gases with elastic collisions can be applied. In particular, in the low density limit, the inelastic Boltzmann equation is derived. Thus, it seems that the basis for this equation is the same as in the elastic case. A different question is the domain in the parameter space of density and coefficient of restitution in which it actually provides an accurate description of the system [4].

The Boltzmann equation is a kinetic equation describing the time evolution of the oneparticle distribution function, that is an average quantity. It is also possible to study, again in the low density limit, fluctuations and correlations in the positions and velocities of the particles by means of the associated distribution functions. It is known that correlations can become quite large in out of equilibrium elastic systems, on the appropriate long time and length scales. This should not be interpreted as a signature of the failure of the Boltzmann equation. The latter only requires that the precollisional part of the correlations of two particles at contact be small $[5,6]$. Closed kinetic equations for the fluctuation and correlation functions, valid in the low density limit, can be derived inside the same approximation schemes that lead to the Boltzmann equation. This includes formal expansions in a small parameter [7] and cluster expansions of the reduced distribution functions [8]. Both methods have been considered for granular gases $[9,10]$. Nevertheless, while the inelastic Boltzmann equation and its implications have been extensively studied, little is known about fluctuations and correlations in granular gases, even though it is widely recognized that they are crucial for the understanding of the properties of these systems.

As mentioned above, granular gases display fluid dynamical behaviour. Therefore, they are expected to obey some kind of hydrodynamic equations, understood as a set of closed equations for the hydrodynamics fields: density, flow velocity, and (granular) temperature. In fact, more or less phenomenological hydrodynamic equations have been successfully applied to many different situations. An appropriate context in which the issue of the existence of hydrodynamics for dilute granular gases, the form of the hydrodynamic equations, and their range of validity, can be addressed is provided by the inelastic Boltzmann equation. By using an extension of the Chapmann-Enskog procedure, the Navier-Stokes equations have been derived, and formal expressions for the transport coefficients obtained. They are, in principle, valid for arbitrary physical values of the coefficient of restitution [11]. Recently, these expressions have been expressed in the form of time integrals of the correlations of some velocity functions computed in a given reference state, the so-called homogeneous cooling state $[12,13]$. Consistency requires that these integrals exist in the long time limit. The above expressions are the generalization of the Green-Kubo relations for elastic gases, although with significative differences that could hardly have been guessed a priori.

It must be stressed that the Chapmann-Enskog method and the Green-Kubo relations presume the existence of hydrodynamics on the appropriate length and time scales and, therefore, they do not add any fundamental support to the validity of a hydrodynamic description for granular gases. On the other hand, if Navier-Stokes equations exist, the Chapmann-Enskog results provide their explicit form. A deeper physical understanding of the structure of the Green-Kubo relations and their validity follows from the analysis of the linearized Boltzmann equation, and more specifically of the spectrum of the associated operator [13]. It is possible to identify the hydrodynamic part of the spectrum, that is the one determining the form of the Green-Kubo relations. It is given by $d+2$ points that in the elastic limit correspond to the $d+2$ collisional invariants, where $d$ is the dimension of the system. The key remaining point for the existence of hydrodynamics is whether the corresponding modes 
dominate for long times and long wavelengths $[14,15]$. Quite interestingly, the hydrodynamic modes also determine the form of the fluctuations of the hydrodynamic quantities in granular systems, reflecting a generalization of the fluctuation-dissipation theorems.

The aim of this paper is to review some recent advances and present some new results along the above ideas. Particular emphasis will be put on the relevance of the hydrodynamic modes for the study of many fundamental and applied issues in granular fluids, as is the case for molecular, elastic systems. In the paper, and especially in the last section, some of the many remaining open questions in the context of the matter discussed here will be indicated.

\section{The homogeneous cooling state of a dilute granular gas}

The system considered is composed of $N$ smooth inelastic hard spheres $(d=3)$ or discs $(d=2)$ of mass $m$ and diameter $\sigma$. Their dynamics consists of free streaming until two particles $i$ and $j$ are in contact, when their velocities change according to the rule

$$
\begin{aligned}
& \boldsymbol{v}_{i} \rightarrow \boldsymbol{v}_{i}^{\prime} \equiv b_{\boldsymbol{\sigma}} \boldsymbol{v}_{i}=\boldsymbol{v}_{i}-\frac{1+\alpha}{2}\left(\hat{\boldsymbol{\sigma}} \cdot \boldsymbol{v}_{i j}\right) \hat{\boldsymbol{\sigma}}, \\
& \boldsymbol{v}_{j} \rightarrow \boldsymbol{v}_{j}^{\prime} \equiv b_{\boldsymbol{\sigma}} \boldsymbol{v}_{j}=\boldsymbol{v}_{j}+\frac{1+\alpha}{2}\left(\hat{\boldsymbol{\sigma}} \cdot \boldsymbol{v}_{i j}\right) \hat{\boldsymbol{\sigma}},
\end{aligned}
$$

where $\boldsymbol{v}_{i j}=\boldsymbol{v}_{i}-\boldsymbol{v}_{j}$ is the relative velocity, $\hat{\boldsymbol{\sigma}}$ is the unit vector pointing from the centre of particle $j$ to the centre of particle $i$ at contact, and $\alpha$ is the coefficient of normal restitution. It is defined in the range $0<\alpha \leqslant 1$ and measures the inelasticity of collisions. Here it will be taken as a constant.

From the above dynamics, the Liouville equation governing the time evolution of the $\mathrm{N}$ particle distribution function of the system can be derived [2,3]. An equivalent representation is given by the BBGKY hierarchy for the $s$-particle distribution functions $(s=1,2, \ldots)$. In the low density limit, a closed equation for the one-particle distribution function, $f(\boldsymbol{r}, \boldsymbol{v}, t)$, can be derived by using the same procedures as for elastic hard particles [2,3]. This is the inelastic Boltzmann equation,

$$
\left(\frac{\partial}{\partial t}+\boldsymbol{v} \cdot \frac{\partial}{\partial \boldsymbol{r}}\right) f(\boldsymbol{r}, \boldsymbol{v}, t)=J[f, f]
$$

where $J[f \mid f]$ is the inelastic Boltzmann collision operator [2, 3, 16-18].

For an isolated granular fluid, no homogenous steady solution is possible. It is trivially seen that the energy of the system decreases monotonically due to the inelasticity of collisions. On the other hand, there is a spatially homogeneous state, the so-called homogeneous cooling state (HCS), for which all the time dependence occurs only through the granular temperature. The latter is defined in the usual way from the average kinetic energy. For the one-particle distribution, this implies a scaling of the form [18]

$$
f_{\mathrm{hcs}}(\boldsymbol{v}, t)=n_{H} v_{0}^{-d}\left[T_{\mathrm{hcs}}(t)\right] \chi_{\mathrm{hcs}}(c), \quad v_{0}\left[T_{\mathrm{hcs}}(t)\right]=\left[\frac{2 k_{\mathrm{B}} T_{\mathrm{hcs}}(t)}{m}\right]^{1 / 2} .
$$

Here $n_{H}$ is the number of particles density, $k_{\mathrm{B}}$ is the Boltzmann constant, and $T_{\mathrm{hcs}}(t)$ is the timedependent temperature of the HCS. Finally, $\chi_{\mathrm{hcs}}(c)$ is an isotropic function of $\boldsymbol{c}=\boldsymbol{v} / v_{0}[T(t)]$. The use of equation (3) in equation (2) provides an integral equation for $\chi_{\mathrm{hcs}}$,

$$
\frac{\zeta_{0}}{2} \frac{\partial}{\partial c} \cdot\left(c \chi_{\mathrm{hcs}}\right)=J_{c}\left[\chi_{\mathrm{hcs}}, \chi_{\mathrm{hcs}}\right]
$$

where $J_{c}$ is the scaled Boltzmann collision operator,

$$
J_{c}\left[\chi_{\mathrm{hcs}}, \chi_{\mathrm{hcs}}\right]=\int \mathrm{d} c_{1} \bar{T}_{0}\left(c, c_{1}\right) \chi_{\mathrm{hcs}}(c) \chi_{\mathrm{hcs}}\left(c_{1}\right),
$$




$$
\bar{T}_{0}\left(\boldsymbol{c}, \boldsymbol{c}_{1}\right)=\int \mathrm{d} \hat{\boldsymbol{\sigma}} \Theta[\boldsymbol{g} \cdot \hat{\boldsymbol{\sigma}}] \boldsymbol{g} \cdot \hat{\boldsymbol{\sigma}}\left[\alpha^{-2} b_{\sigma}^{-1}\left(\boldsymbol{c}, \boldsymbol{c}_{1}\right)-1\right],
$$

with $\boldsymbol{g} \equiv \boldsymbol{c}-\boldsymbol{c}_{1}, \Theta$ the Heaviside step function, and $b_{\sigma}^{-1}$ an operator replacing the velocities $c$ and $c_{1}$ to its right by the precollisional values obtained by inverting the collision rules given in equation (1). Moreover, the scaling property (3) implies that the time dependence of the temperature is given by the Haff law:

$$
T_{\mathrm{hcs}}(t)=T_{\mathrm{hcs}}(0)\left[1+\frac{1}{2} \zeta_{\mathrm{hcs}}(0) t\right]^{-2} \text {. }
$$

The cooling rate of the HCS, $\zeta_{\text {hcs }}(t)$, is given by

$$
\zeta_{\mathrm{hcs}}(t)=\frac{\left(1-\alpha^{2}\right) \pi^{\frac{d-1}{2}} \sigma^{d-1} n_{H} v_{0}(t)}{2 \Gamma\left(\frac{d+3}{2}\right) d} \int \mathrm{d} c \int \mathrm{d} c_{1} g^{3} \chi_{\mathrm{hcs}}(c) \chi_{\mathrm{hcs}}\left(c_{1}\right),
$$

and

$$
\zeta_{0}=\frac{\ell \zeta_{\mathrm{hcs}}(t)}{v_{0}\left[T_{\mathrm{hcs}}(t)\right]}
$$

where $\ell=1 / n_{H} \sigma^{d-1}$ is proportional to the mean free path of the particles. Since $\zeta_{\text {hcs }} \propto T_{\mathrm{hcs}}^{1 / 2}$, equation (7) becomes independent of the initial condition in the long time limit,

$$
T_{\mathrm{hcs}} \sim 4(\bar{\zeta} t)^{-2}, \quad \bar{\zeta}=\frac{\zeta_{\mathrm{hcs}}(t)}{T_{\mathrm{hcs}}^{1 / 2}(t)},
$$

indicating that all the homogeneous cooling states of a given system tend to converge in the long time limit [30]. This seems to suggest the existence of some kind of $H$ theorem for the homogeneous Boltzmann equation for inelastic hard particles, although no proof of such a theorem has been given to date.

\section{Linearized Boltzmann equation and hydrodynamic modes}

Now, the initial value problem for small perturbations around the HCS of an isolated granular gas will be considered. In this case, the Boltzmann equation can be linearized around the HCS, and it will be shown that, in appropriate dimensionless units, its general solution can be related to a linear eigenvalue problem. By considering times over which the deviation of the distribution function from $f_{\text {hcs }}$ remains small, we can write

$$
f(\boldsymbol{r}, \mathbf{v}, t)=f_{\mathrm{hcs}}(\boldsymbol{v}, t)+\delta f(\boldsymbol{r}, \mathbf{v}, t), \quad|\delta f(\boldsymbol{r}, \boldsymbol{v}, t)| \ll f_{\mathrm{hcs}}(\boldsymbol{v}, t) .
$$

In order to eliminate the time dependence of the HCS, it is convenient to introduce the scaled velocity $c$ defined below equation (3) and the dimensionless quantities

$\boldsymbol{l}=\frac{\boldsymbol{r}}{\ell}, \quad s=\int_{0}^{t} \mathrm{~d} t^{\prime} \frac{v_{0}\left(t^{\prime}\right)}{\ell}, \quad \delta \chi(\boldsymbol{l}, \boldsymbol{c}, s)=n_{H}^{-1} v_{0}^{d}(t) \delta f(\boldsymbol{r}, \boldsymbol{v}, t)$.

The time scale $s$ is proportional to the average of the accumulated number of collisions per particle in the interval $(0, t)$. Then, substitution of equation (11) into the Boltzmann equation, retaining only terms up to first order in the deviations, gives the linear Boltzmann equation

$$
\frac{\partial}{\partial s} \delta \chi+c \cdot \frac{\partial}{\partial \boldsymbol{l}} \delta \chi=\Lambda \delta \chi(\boldsymbol{l}, \boldsymbol{c}, s)
$$

where the linear operator $\Lambda$ is given by

$$
\Lambda \delta \chi=J_{c}\left[\chi_{\mathrm{hcs}}, \delta \chi\right]+J_{c}\left[\delta \chi, \chi_{\mathrm{hcs}}\right]-\frac{\zeta_{0}}{2} \frac{\partial}{\partial \boldsymbol{c}} \cdot(\boldsymbol{c} \delta \chi) .
$$


In this representation, the effect of the cooling appears explicitly through the last term on the right-hand side of the above expression.

Solutions to equation (13) will be sought in a Hilbert space defined by the scalar product

$$
\langle g \mid h\rangle=\int \mathrm{d} \mathbf{c} \chi_{\mathrm{hcs}}^{-1}(c) g^{*}(\mathbf{c}) h(\mathbf{c}),
$$

where $g^{*}$ denotes the complex conjugate of $g$. It must be noted that this is a stronger requirement than the necessary normalization of the distribution function. Since the collision operator $\Lambda$ does not affect the space variable, it is enough to consider a single Fourier mode, i.e.,

$$
\delta \chi(l, c, s)=\mathrm{e}^{\mathrm{i} k \cdot l} \delta \tilde{\chi}(\boldsymbol{k}, \boldsymbol{c}, s),
$$

so that the linearized Boltzmann equation becomes

$$
\frac{\partial}{\partial s} \delta \tilde{\chi}=(\Lambda-\mathrm{i} k \cdot c) \delta \tilde{\chi}
$$

All linear excitations are determined from the spectrum of the operator $\Lambda-\mathrm{i} k \cdot c$. The hydrodynamic part of this spectrum is defined as follows. For $k=0$ it is given by those eigenvalues that coincide with the eigenvalues of the exact balance equations for the momentum density, flow velocity, and temperature obtained from the linearized homogeneous Boltzmann equation, in the same limit $k=0$, i.e., for homogeneous states. Assuming that the cooling rate for homogeneous systems can be expressed as a function of the density and the temperature, these eigenvalues are found to be [13]

$$
\lambda_{1}=0, \quad \lambda_{2}=\zeta_{0} / 2 \quad \lambda_{3}=-\zeta_{0} / 2,
$$

the eigenvalue $\lambda_{2}$ being $d$-fold degenerated. For finite $k$, the hydrodynamic modes are defined as the solutions of the equation

$$
(\Lambda-\mathrm{i} k \cdot c) \xi_{i}(\boldsymbol{k}, \mathbf{c})=\lambda_{i} \xi_{i}(\boldsymbol{k}, \mathbf{c})
$$

that are continuously connected as functions of $k$ to the above special solutions for $k=0$. Of course, consistency requires that $\Lambda$ includes the spectrum of the balance equations for $k=0$. Straightforward calculations show that [13]

$$
\Lambda \xi_{i}(c)=\lambda_{i} \xi_{i}(c), \quad i=1,2,3
$$

with the $\lambda_{i}$ given by equation (18) and

$$
\begin{aligned}
& \xi_{1}(c)=\chi_{\mathrm{hcs}}(c)+\frac{\partial}{\partial c} \cdot\left[c \chi_{\mathrm{hcs}}(c)\right], \\
& \xi_{2}(c)=-\frac{\partial \chi_{\mathrm{hcs}}(c)}{\partial c}, \quad \xi_{3}(c)=-\frac{\partial}{\partial c} \cdot\left[c \chi_{\mathrm{hcs}}(c)\right] .
\end{aligned}
$$

This confirms the existence of the hydrodynamic modes. Their evaluation for finite $k$ is now a technical problem. To Navier-Stokes order, they have been computed by using perturbation expansion methods [14], and the results agree with those obtained from the Navier-Stokes equations derived by means of the Chapmann-Enskog procedure [11]. Here an alternative approach, designed to derive in a direct way expressions for the Navier-Stokes transport coefficients, will be outlined.

As a consequence of $\Lambda$ being not Hermitian, the eigenfunctions $\xi_{i}$ are not orthogonal. It is then useful to introduce a set of functions $\left\{\bar{\xi}_{i}\right\}$ that are biorthogonal to the set $\left\{\xi_{i}, i=1,2,3\right\}$. A convenient choice is

$$
\left\{\bar{\xi}_{i}\right\}=\left\{\chi_{\mathrm{hcs}}(c), c \chi_{\mathrm{hcs}}(c),\left(\frac{c^{2}}{d}+\frac{1}{2}\right) \chi_{\mathrm{hcs}}(c)\right\}, \quad\left\langle\bar{\xi}_{i} \mid \xi_{j}\right\rangle=\delta_{i, j}
$$


The general solution to the linearized Boltzmann equation in the Hilbert space can be formally written as

$$
\delta \tilde{\chi}(\boldsymbol{k}, \boldsymbol{c}, s)=\sum_{i} \tilde{a}_{i}(\boldsymbol{k}, s) \chi_{i}(\boldsymbol{k}, \boldsymbol{c})+\delta \tilde{\chi}^{m}(\boldsymbol{k}, \boldsymbol{c}, s),
$$

where the sum extends over the $d+2$ hydrodynamic modes and the term $\delta \tilde{\chi}^{m}$ contains all the other 'microscopic' excitations. Assuming that the hydrodynamic spectrum dominates for long times and small gradients, $\delta \tilde{\chi}^{m}$ can be neglected, and the coefficients $\tilde{a}_{i}$ are given by

$$
\tilde{a}_{i}(\boldsymbol{k}, s)=\left\langle\bar{\xi}_{i} \mid \delta \tilde{\chi}(\boldsymbol{k}, s)\right\rangle,
$$

since $\delta \tilde{\chi}$ now lies in the subspace spanned by the eigenfunctions $\left\{\xi_{i}\right\}$. These coefficients are combinations of the hydrodynamic fields, i.e., they can be expressed in terms of the deviations of the macroscopic density $n$, temperature $T$ and fluid velocity $\boldsymbol{u}$. It is now possible to derive a formal expression for $\delta \tilde{\chi}(k, c, s)$ to first order in $k$, and from it the Navier-Stokes order for the pressure tensor and the heat conductivity [13]. The results will be given in the next section.

The above discussion relies on the assumption that the hydrodynamic point spectrum found here is isolated and smaller than all the other parts of the spectrum, so that it dominates for long times and long wavelengths. This is a necessary condition for the existence of hydrodynamics and has been proven in the elastic case. The proof does not extend directly to the inelastic case, although there are some reasons to expect that this is the case even for rather strong inelasticity. A detailed discussion of the basis for a hydrodynamic description of a granular gas in this context is given in [15].

\section{Green-Kubo representation of the transport coefficients}

The procedure described in the previous section leads to the following expressions for the dissipative part of the pressure tensor $\Pi_{i j}(\boldsymbol{r}, t)$ and the heat flux $\boldsymbol{q}(\boldsymbol{r}, t)$ to Navier-Stokes order [13]:

$$
\Pi_{i j}=-\eta\left(\frac{\partial u_{i}}{\partial r_{j}}+\frac{\partial u_{j}}{\partial r_{i}}-\frac{2}{d} \delta_{i j} \nabla \cdot \mathbf{u}\right), \quad \boldsymbol{q}=-\kappa \nabla T-\mu \nabla n,
$$

where $\eta$ is the shear viscosity, $\kappa$ the (thermal) heat conductivity, and $\mu$ a new transport coefficient that vanishes in the elastic limit and that will be referred to as the diffusive heat conductivity. They are given by

$$
\begin{aligned}
& \eta=n_{H} m \ell v_{0}(T) \int_{0}^{\infty} \mathrm{d} s \mathrm{e}^{-s \zeta_{0} / 2}\left\langle\Delta_{x y}(s) \Phi_{2, x y}\right\rangle, \\
& \kappa=n_{H} \ell v_{0}(T) \int_{0}^{\infty} \mathrm{d} s \mathrm{e}^{s \zeta_{0} / 2}\left\langle\Sigma_{x}(s) \Phi_{3, x}\right\rangle \\
& \mu=m \ell v_{0}^{3}(T)\left[\int_{0}^{\infty} \mathrm{d} s\left(\mathrm{e}^{s \zeta_{0} / 2}-1\right)\left\langle\Sigma_{x}(s) \Phi_{3, x}\right\rangle+\frac{1}{2} \int_{0}^{\infty} \mathrm{d} s\left\langle\Sigma_{x}(s) c_{x}\right\rangle\right] .
\end{aligned}
$$

In the above expressions, $\Delta_{x y}$ and $\Sigma_{x}$ are the transversal momentum flux and the heat flux, respectively,

$$
\Delta_{x y}(c)=c_{x} c_{y}, \quad \Sigma_{x}(c)=\left(c^{2}-\frac{d+2}{2}\right) c_{x},
$$

while $\Phi_{2, i j}$ and $\Phi_{3, x}$ are 'modified fluxes',

$$
\Phi_{2, x y}(c)=-c_{x} \frac{\partial \ln \chi_{\mathrm{hcs}}(c)}{\partial c_{y}}, \quad \Phi_{3, x}(c)=-\frac{c_{x}}{2}\left[d+c \cdot \frac{\partial \ln \chi_{\mathrm{hcs}}(c)}{\partial c}\right] .
$$


The angular brackets denote 'time-correlation functions' in the HCS,

$$
\langle g(s) h\rangle \equiv \int \mathrm{d} c \chi_{\mathrm{hcs}}(c) g(c, s) h(c),
$$

where the time dependence is given by

$$
g(c, s)=\mathrm{e}^{s \tilde{\Lambda}(c)} g(c),
$$

with

$\tilde{\Lambda}(\mathbf{c})=\int \mathrm{d} \boldsymbol{c}_{1} \chi_{\mathrm{hcs}}\left(c_{1}\right) \int \mathrm{d} \hat{\boldsymbol{\sigma}} \Theta(\boldsymbol{g} \cdot \hat{\boldsymbol{\sigma}}) \mathbf{g} \cdot \hat{\boldsymbol{\sigma}}\left[b_{\sigma}\left(\boldsymbol{c}, \boldsymbol{c}_{1}\right)-1\right](1+P)+\frac{\zeta_{0}}{2} \boldsymbol{c} \cdot \frac{\partial}{\partial \boldsymbol{c}}$.

Here, $P$ is an operator that interchanges the velocities $c$ and $c_{1}$ to its right. Equations (26)(28) are the generalization for inelastic hard collisions of the dilute limit of the well-known Green-Kubo relations for molecular fluids, reducing to them in the elastic limit [12]. They can be understood as generalized fluctuation-dissipation relations, in the sense that they relate the transport coefficients characterizing dissipation in inhomogeneous systems with the time decay of fluctuations in the homogeneous reference state (the HCS). Equivalent expressions for the transport coefficients are obtained by solving the nonlinear Boltzmann equation by the Chapman-Enskog method $[11,12]$ and also by perturbation analysis of the hydrodynamic spectrum of the linear Boltzmann equation [14]. The effect of the inelasticity of collisions manifests itself in several ways. Perhaps the most unexpected among them is that the initial dissipative fluxes $\Delta_{x y}$ and $\Sigma_{x}$ are replaced by the modified fluxes $\Phi_{2, x y}$ and $\Phi_{3, x}$ in the time correlation functions appearing in the expressions of $\eta$ and $\kappa$. This is a direct consequence of the form of the hydrodynamic modes. While in a molecular system they are given by linear combinations of the Maxwellian times $1, c$, and $c^{2}$, in granular gases they are given by equation (21), and it should be realized that $\chi_{\mathrm{hcs}}(c)$ is not a Maxwellian outside the elastic limit $\alpha=1$.

Since the diffusive heat conductivity $\mu$ is a peculiarity of granular systems, it seems appropriate to comment about its relevance. In general, its contribution to the heat flux is much smaller than the one coming from the usual heat conductivity associated with the temperature gradient, because $\kappa \gg \mu$ for all $\alpha$. Nevertheless, there are some cases where the existence of this contribution manifests itself in a clear way. Consider, for instance, an open granular system in the presence of gravity. The system is fluidized by means of a vibrating bottom plate. In the steady state, the granular temperature profile presents a minimum at a certain height, increasing from there on. This temperature inversion has been observed in computer simulations [19-21] and also in experiments [22-25]. Its theoretical explanation is tied to the existence of the diffusive contribution to the heat flux [19], and the value of the transport coefficient $\mu$ can be directly obtained from the measurement of the heat flux at the temperature minimum [26]. For a low density gas, the results are in good agreement with the theoretical predictions discussed in the next section.

\section{Simulation study of the Green-Kubo relations}

The formal expressions of the transport coefficients given by equations (26)-(28) are rather involved and have been evaluated analytically only in some approximations, namely by introducing an expansion in orthogonal functions, keeping only rather arbitrarily the lowest orders contributions $[11,27,28]$. On the other hand, the above Green-Kubo relations can be transformed into a form that is suitable for evaluation by means of $N$-particle simulation techniques. Two main points must be addressed for that. First, there is the technical difficulty 

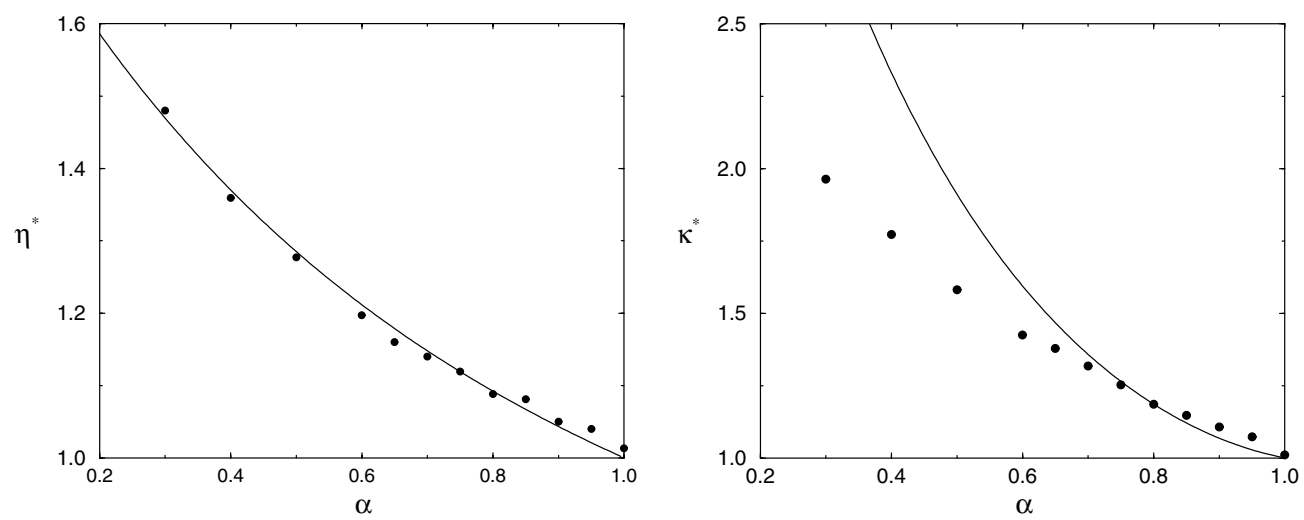

Figure 1. Left: the dimensionless reduced coefficient of shear viscosity $\eta^{*}$ as a function of $\alpha$. The symbols are from the DSMC method, while the solid curve is the analytical result obtained in the first Sonine approximation [11]. Right: the same for the reduced coefficient of thermal heat conductivity $\kappa^{*}$.

that the operator $\tilde{\Lambda}$, defining the time dependence of the fluxes, involves the cooling rate $\zeta_{0}$ that, therefore, should be known a priori, and it is not. This can be overcome by making a change in the time scale and realizing the existence of an exact mapping of the HCS onto a steady state [29, 30]. The second issue is to transform equations (26)-(28) into expressions involving a well-defined $N$-particle dynamics, instead of the effective Boltzmann dynamics defined by $\tilde{\Lambda}$. This can be done by using the same kind of assumptions as are needed to derive the Boltzmann equation and, in particular, assuming that velocity correlations in the HCS are negligible in the low density limit [30].

We have used the direct simulation Monte Carlo (DSMC) method [31] to simulate the $N$-particle dynamics of a dilute granular gas composed of inelastic hard spheres $(d=3)$. The details of the practical implementation of the above ideas and the simulation technique are discussed in [30] and [32]. Here it will only be stressed that the DSMC method allows us to simulate a homogeneous state in such a way that it stays homogeneous 'by definition', avoiding the spontaneous developing of spatial inhomogeneities. This is important in the present context, since the HCS is known to be unstable against spatial long wave perturbations, even in the low density limit. This is the so-called clustering instability [33, 34].

In the simulations, it is observed that the HCS, or more precisely its equivalent steady representation, is reached after a few collisions per particle. This supports that the hydrodynamic point spectrum identified in section 4 is isolated and smaller than all the other parts of the spectrum. Moreover, all the time correlation functions appearing in equations (26)(28) are found to decay exponentially, at least until they decay by two orders of magnitude from their initial values. This has been exploited in order to compute the time integrals. The results obtained for the shear viscosity are shown in figure 1 (left). What is actually plotted is the shear viscosity scaled with its elastic value in the first Sonine approximation, i.e.,

$$
\eta^{*} \equiv \frac{\eta(T)}{\eta_{0}(T)}, \quad \eta_{0}(T)=\frac{5\left(m k_{\mathrm{B}} T\right)^{1 / 2}}{16 \pi^{1 / 2} \sigma^{2}} .
$$

Also plotted in the same figure is the approximate theoretical prediction obtained when computing equation (26) in the first Sonine approximation [11]. It is observed that the viscosity monotonically increases with the inelasticity of collisions (decreasing $\alpha$ ) and that the theoretical prediction is in good agreement with the simulation results over all the range of values of $\alpha$. 


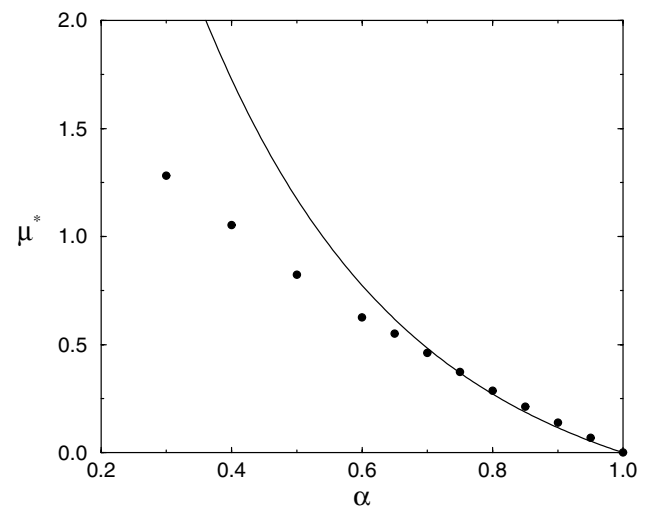

Figure 2. The same as in figure 1, but for the reduced coefficient of diffusive heat conductivity $\mu^{*}$.

The simulation results for the (thermal) heat conductivity are given in figure 1 (right). Similarly to above, the dimensionless plotted quantity is

$$
\kappa^{*} \equiv \frac{\kappa(T)}{\kappa_{0}(T)}, \quad \kappa_{0}(T)=\frac{75 k_{\mathrm{B}}\left(k_{\mathrm{B}} T\right)^{1 / 2}}{64(\pi m)^{1 / 2} \sigma^{2}} .
$$

Again, it is found that the transport coefficient is a monotonic decreasing function of $\alpha$. However, in this case the first Sonine approximation leads to significant quantitative discrepancies for small values of $\alpha$. This may be, at least partially, due to the fact that the fluxes appearing in the expression of the heat conductivity involve higher velocity moments than those present in the expression of the shear viscosity.

The dimensionless reduced diffusive heat conductivity $\mu^{*}$ has been defined as

$$
\mu^{*} \equiv \frac{n \mu(T)}{T \kappa_{0}(T)},
$$

and the results obtained for it are presented in figure 2. As is the case for the thermal heat conductivity, the first Sonine approximation significantly overestimates this transport coefficient for strong dissipation.

The analysis of the transport coefficients for a dilute gas of inelastic hard discs $(d=2)$ has been reported in [32]. The results are quite similar, the main difference being that the discrepancies of the first Sonine approximation predictions are stronger for $d=2$ than for $d=3$, as is usually the case.

As already pointed out, in order to transform the effective one-particle dynamics, implicit in the linearized evolution operator $\tilde{\Lambda}$, into an $N$-particle dynamics, it has been assumed that velocity correlations are negligible in the HCS of a low density granular gas. Nevertheless, when the DSMC data are carefully analysed, it is seen that for strong inelasticity $(\alpha \lesssim 0.6)$, velocity correlation effects are clearly identified [32]. For instance, their contribution to the initial value of the time-correlation functions appearing in the $N$-particle representation of the Green-Kubo expressions can be up to $10 \%$ of the total value. This raises some fundamental issues. Of course, a first possibility is that the velocity correlations are just an artefact introduced by the simulation method itself, i.e., an inaccuracy of the DSMC method. On the other hand, if they are not, how can these correlations be incorporated in a theoretical description of dilute granular gases? Is it consistent with the description provided by the inelastic Boltzmann equation? A partial answer to these questions will be given in the next section. 


\section{Velocity correlations and total energy fluctuations in the HCS}

In spite of the great advance in the understanding of rapid granular flows in recent years, little is known about fluctuations and correlations in those systems. Most previous studies have focused on the study of the initial build-up of spatial correlations in the development of the clustering instability $[35,36]$, or on the short time and length scale precollisional velocity correlations when starting from a thermal equilibrium state [37]. Recently, an empirical expression for the two-particle distribution function of a system in the HCS, based on simplicity and symmetry considerations, has been proposed [38]. It includes both spatial and velocity correlations, but their relation with the dynamics of the system has not been analysed.

Closed equations for the distribution functions characterizing correlations in a dilute granular gas can be derived by using the same kind of approximations as are needed to derive the inelastic Boltzmann equation. In particular, in the hierarchy method it is assumed that the order in the density of the precollisional part of the correlation functions of two particles in contact increases as the number of involved particles increases [8]. Of course, the results obtained under this assumption must be consistent with it.

Here we will restrict ourselves to a dilute gas in the HCS. It is assumed that the Liouville equations admits a solution describing this state and having the scaling form

$$
\rho_{\mathrm{hcs}}\left(\left\{\boldsymbol{r}_{i}, \boldsymbol{v}_{i}\right\}, t\right)=\left[v_{0}(t)\right]^{-d N} \rho_{\mathrm{hcs}}^{*}\left(\left\{\boldsymbol{r}_{i j}\right\},\left\{\boldsymbol{c}_{i}\right\}\right),
$$

that is invariant under space translations. This implies an analogous scaling for all the distribution functions of the system and, in particular, leads to equation (3) for the oneparticle distribution function. Two-particle correlations are described by means of the function $g_{\text {hcs }}\left(\boldsymbol{r}_{12}, \boldsymbol{v}_{1}, \boldsymbol{v}_{2}, t\right)$, defined through the cluster expansion of the two-particle distribution function $f_{2, \text { hcs }}\left(\boldsymbol{r}_{12}, \boldsymbol{v}_{1}, \boldsymbol{v}_{2}, t\right)$,

$$
f_{2, \mathrm{hcs}}\left(\boldsymbol{r}_{12}, \boldsymbol{v}_{1}, \boldsymbol{v}_{2}, t\right)=f_{\mathrm{hcs}}\left(\boldsymbol{v}_{1}, t\right) f_{\mathrm{hcs}}\left(\boldsymbol{v}_{2}, t\right)+g_{\mathrm{hcs}}\left(\boldsymbol{r}_{12}, \boldsymbol{v}_{1}, \boldsymbol{v}_{2}, t\right) .
$$

As a consequence of the scaling property (37), the reduced distribution function

$$
\tilde{g}_{\text {hcs }}\left(\boldsymbol{l}_{12}, \boldsymbol{c}_{1}, \boldsymbol{c}_{2}\right)=n_{H}^{-1} \ell^{d} v_{0}^{2 d}(t) g_{\mathrm{hcs}}\left(\boldsymbol{r}_{12}, \boldsymbol{v}_{1}, \boldsymbol{v}_{2}, t\right)
$$

does not depend on $s$. Then, from the second equation of the BBGKY hierarchy, neglecting the term involving three-particle correlations, a closed equation for $\tilde{g}_{\text {hcs }}$ is found [10],

$$
\left[\boldsymbol{c}_{12} \cdot \frac{\partial}{\partial \boldsymbol{l}_{12}}-\Lambda\left(\boldsymbol{c}_{1}\right)-\Lambda\left(\boldsymbol{c}_{2}\right)\right] \tilde{g}_{\mathrm{hcs}}\left(\boldsymbol{l}_{12}, \boldsymbol{c}_{1}, \boldsymbol{c}_{2}\right)=\delta\left(\boldsymbol{l}_{12}\right) \bar{T}_{0}\left(\boldsymbol{c}_{1}, \boldsymbol{c}_{2}\right) \chi_{\mathrm{hcs}}\left(c_{1}\right) \chi_{\mathrm{hcs}}\left(c_{2}\right) \text {. }
$$

In order to focus on the velocity correlations, we introduce the marginal velocity correlation function

$$
\psi_{\mathrm{hcs}}\left(c_{1}, c_{2}\right) \equiv \int \mathrm{d} l_{12} \tilde{g}_{\mathrm{hcs}}\left(\boldsymbol{l}_{12}, c_{1} ; c_{2}\right)+\delta\left(c_{1}-c_{2}\right) \chi_{\mathrm{hcs}}\left(c_{1}\right)
$$

Integration of equation (40) yields

$$
\left[\Lambda\left(c_{1}\right)+\Lambda\left(c_{2}\right)\right] \psi_{\mathrm{hcs}}\left(c_{1}, c_{2}\right)=-\bar{T}_{0}\left(c_{1}, c_{2}\right) \chi_{\mathrm{hcs}}\left(c_{1}\right) \chi_{\mathrm{hcs}}\left(c_{2}\right) .
$$

Trying to find the complete solution of the above equation is a formidable task. We will restrict ourselves to finding the hydrodynamic part $\psi_{\mathrm{hcs}}^{(h)}$ of the solution, defined by

$$
\psi_{\mathrm{hcs}}^{(h)}\left(\boldsymbol{c}_{1}, \boldsymbol{c}_{2}\right) \equiv \mathcal{P} \psi_{\mathrm{hcs}}\left(\boldsymbol{c}_{1}, \boldsymbol{c}_{2}\right)
$$

where $\mathcal{P}$ is the projection operator onto the direct product of the hydrodynamic part of Hilbert space. Next, we apply $\mathcal{P}$ to both sides of equation (42) and, in order to get a closed equation for $\psi_{\text {hcs }}^{(h)}$, we make the approximation $\mathcal{P} \Lambda\left(c_{i}\right)=\mathcal{P} \Lambda\left(c_{i}\right) \mathcal{P}$. Then, the resulting equation can be solved yielding [10]

$$
\psi_{\mathrm{hcs}}^{(h)}\left(\boldsymbol{c}_{1}, \boldsymbol{c}_{2}\right)=a_{33}(\alpha) \xi_{3}\left(\boldsymbol{c}_{1}\right) \xi_{3}\left(\boldsymbol{c}_{2}\right) .
$$



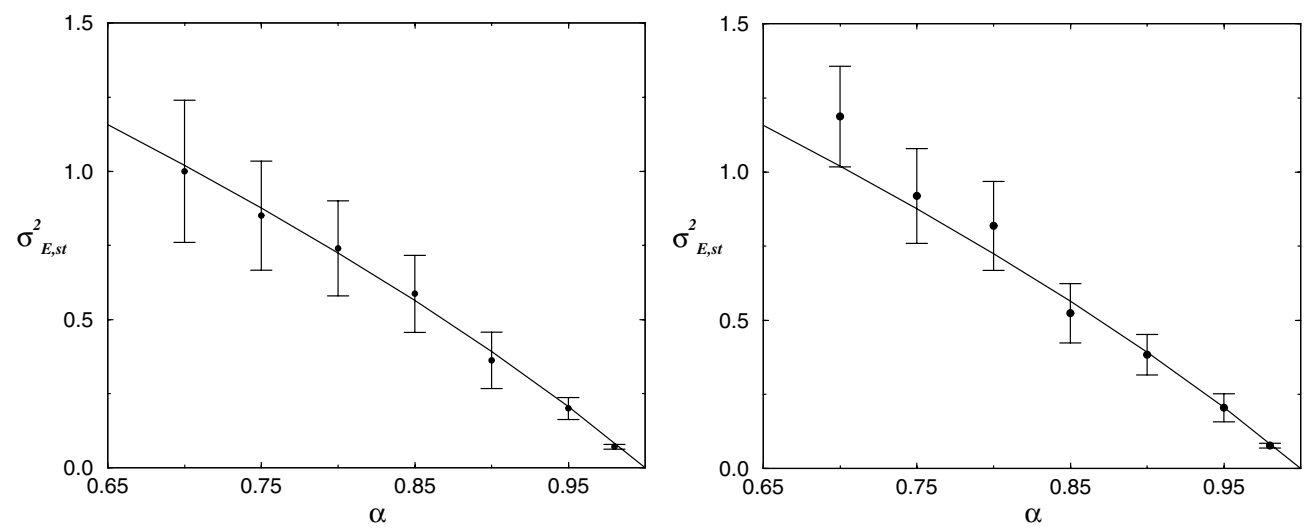

Figure 3. Scaled second moment of the energy fluctuations $\sigma_{E, s t}^{2}$ as a function of the restitution coefficient $\alpha$. The symbols are from MD simulations of a system of hard discs and the solid curve the theoretical prediction given by equation (45). Left: $n_{H}=5 \times 10^{-3} \sigma^{-2}$; right: $n_{H}=0.01 \sigma^{-2}$.

The coefficient $a_{33}(\alpha)$ is a functional of $\chi_{\mathrm{hcs}}$, vanishing in the limit $\alpha \rightarrow 1$. An explicit expression can be obtained in the first Sonine approximation [10]. The fact that there are no contributions to $\psi_{\mathrm{hcs}}^{(h)}$ from the other combinations of the hydrodynamic modes is a consequence of the symmetry of the HCS and the strict conservation of the number of particles and the total momentum. On the other hand, equation (44) clearly shows the presence of velocity correlations that are due to the inelasticity of collision being, therefore, inherent to granular systems.

A direct test of the accuracy of the above expression by means of $N$-particle simulation is beyond present possibilities. Nevertheless, it is possible to carry out an indirect check by computing the fluctuations of the total energy $E$. They are given by

$$
\begin{aligned}
\left\langle[\delta E(t)]^{2}\right\rangle_{\mathrm{hcs}} & \equiv\left\langle E^{2}(t)\right\rangle_{\mathrm{hcs}}-\langle E(t)\rangle_{\mathrm{hcs}}^{2} \\
& =N k_{\mathrm{B}}^{2} T_{\mathrm{hcs}}^{2}(t) \int \mathrm{d} c_{1} \int \mathrm{d} c_{2} c_{1}^{2} c_{2}^{2} \psi_{\mathrm{hcs}}^{(h)}\left(c_{1}, c_{2}\right) \\
& =N k_{\mathrm{B}}^{2} T_{\mathrm{hcs}}^{2}(t) e(\alpha),
\end{aligned}
$$

with $e(\alpha)=d^{2} a_{33}(\alpha)$. In the above expressions, $\langle\cdots\rangle_{\text {hcs }}$ denotes ensemble average with $\rho_{\mathrm{hcs}}\left(\left\{\boldsymbol{r}_{i}, \boldsymbol{v}_{i}\right\}, t\right)$. It is seen that the energy fluctuations are proportional to the size of the system $N$, as expected from the central limit theorem. Besides, they vanish in the elastic limit since we are considering an isolated system, that corresponds to the microcanonical ensemble for $\alpha=1$.

To put the above expression in a proper context, it is important to realize that, although its derivation relies on the knowledge of the hydrodynamic modes, the validity of the result is not restricted to any kind of hydrodynamic regime. On the contrary, it is a general result for the HCS, assuming it exists, with the only operational approximation given above equation (44). We have measured the energy fluctuations by performing molecular dynamics (MD) simulations of a freely evolving system of inelastic hard discs. Periodic boundary conditions were used, and the system size was always chosen smaller than the critical value needed for the spontaneous formation of spatial instabilities [33]. The results obtained are given in figure 3 for two small densities, namely $n_{H}=5 \times 10^{-3} \sigma^{-2}$ and $0.01 \sigma^{-2}$. The plotted quantity is $\sigma_{E, s t}^{2} \equiv\left\langle(\delta E)^{2}\right\rangle_{\mathrm{hcs}} / N k_{\mathrm{B}}^{2} T_{\mathrm{hcs}}^{2}$, which does not depend on time, and each of the reported values corresponds to an average over several trajectories. Also plotted is 


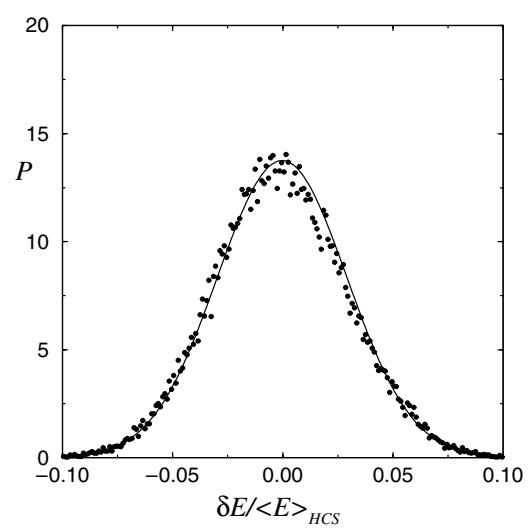

Figure 4. Normalized distribution of the relative energy fluctuations for a system of inelastic hard disc with $n_{H}=0.02 \sigma^{-2}$. The symbols are from MD simulations and the solid curve is a fit to a Gaussian.

the theoretical prediction provided by equation (45), i.e., the function $e(\alpha)$ computed in the first Sonine approximation. A quite good agreement is observed over all the range of $\alpha$ considered, specially at the lowest density. This was expected, given the low density character of the theoretical approach sketched above. Unfortunately, considering smaller values of the restitution coefficient requires the simulations of too small systems in order to avoid the instability mentioned above, and consequently finite size effects may become relevant.

Then, we conclude that there are intrinsic velocity correlations in a granular fluid as a consequence of the inelasticity of collisions, even in the low density limit. Moreover, they increase as the value of $\alpha$ decreases, as expected. Also, the results in figure 3 indicate that what we have termed the hydrodynamic part of the velocity correlations is well described by a consistent low density theory, at least for $\alpha \gtrsim 0.7$. On the other hand, it is not possible to infer anything more precise from the results in this section with regard to the manifestation of velocity correlation effects in the evaluation of the Green-Kubo expressions, as discussed in the previous section. There are two reasons for this: (1) it is easily realized that the velocity correlations involved in the Green-Kubo expressions lie outside the hydrodynamic subspace, and (2) as already indicated, their effect become appreciable for $\alpha \lesssim 0.6$, i.e., for systems more inelastic than those considered in the MD simulations reported here.

From the MD simulation results, it is also possible to compute the normalized distribution of the scaled energy fluctuations $\delta E /\langle E\rangle_{\text {hcs }}$. An example, corresponding to $\alpha=0.8$ and $n_{H}=0.02 \sigma^{-2}$, is shown in figure 4 . The results are well fitted by a Gaussian, within the numerical precision of the data. In particular, no asymmetry is observed. To date, there is no theoretical explanation available for this simple behaviour in an inherent non-equilibrium system.

\section{Discussion}

In this paper, several questions related to the description of dilute granular gases have been addressed. The basic theoretical ingredient for the discussion has been the identification of the hydrodynamic part of the spectrum of the linearized Boltzmann equation for smooth inelastic hard spheres or discs. In the limit of vanishing wavevector, the hydrodynamic modes are determined in a unique way by the exact balance equations for the density, momentum, 
and energy, for all values of the coefficient of restitution. Beyond this limit, one has to resort to some approximations, for instance perturbation expansions, assuming analyticity and convergence. Moreover, if the hydrodynamic modes dominate for long times and long wavelengths, the hydrodynamic Navier-Stokes equations for a dilute granular gas follow, as discussed in section 3. Therefore, the fundamental and controversial question of the existence of a hydrodynamic description for granular gases described by the Boltzmann equation can be sharply formulated as: are the hydrodynamic eigenvalues smaller than all the other parts of the spectrum? For elastic systems, this question has been positively answered [39], and it can be expected that much more will be learned about the spectrum of the inelastic linear Boltzmann equation in the near future.

The procedure indicated above leads to formal expressions for the transport coefficients of a dilute granular gas that can be expressed in the form of Green-Kubo relations. The energy dissipation in collisions introduces significant differences as compared with the usual relations for molecular, elastic gases. The expressions have been evaluated by means of an $N$-particle simulation technique. The results are in quite good agreement with the analytical expressions obtained in the first Sonine approximation for not too strongly inelastic systems, but significant discrepancies are observed for small values of the coefficient of restitution. An analysis of the simulation data shows that they are, at least in part, due to the presence of relevant velocity correlations in the HCS, even in the low density limit. To what extent this implies that the effect of velocity correlations should be incorporated somehow in the theoretical analysis of the transport coefficients of a dilute granular gas is an open question. A related question is the role of velocity correlations for finite density. It has been found that velocity correlations might become important somewhat before a granular system develops significant short range spatial correlations [4]. Therefore, extensions of the Boltzmann equation to finite density should include the effect of velocity correlations in the precollisional sphere, in addition to the spatial correlations. This offers another challenge to the researchers on granular fluids.

As a necessary first step in the analysis of the velocity correlations in the HCS of granular gases, a distribution function partially describing their presence has been computed. Again, advantage has been taken of the knowledge of the hydrodynamic modes for a vanishing wavevector. The identified velocity correlations have an intrinsic inelastic character, vanishing in the elastic limit, although the theoretical analysis carried out predicts that they stay small for all values of the restitution coefficient. The presence of velocity correlations implies that the total energy of the system fluctuates. We have compared the theoretical predictions for these fluctuations with molecular dynamics results and found a good agreement. Nevertheless, due to limitations associated with the instability of the HCS, the simulation results are restricted to not very strong inelasticity. The issue of whether the theory also works in the very small $\alpha$ region deserves future work.

\section{Acknowledgments}

This research was supported in part by the Ministerio de Ciencia y Tecnología (Spain) through Grant No. BFM2002-00307 (partially financed by FEDER funds).

\section{References}

[1] Brilliantov N V and Pöschel T 2004 Kinetic Theory of Granular Gases (Oxford: Oxford University Press)

[2] Brey J J, Dufty J W and Santos A 1997 J. Stat. Phys. 871051

[3] van Noije T P C, Ernst M and Brito R 1998 Physica 251266

[4] Brey J J and Ruiz-Montero M J 2004 Phys. Rev. E 69011305 
[5] Karkheck J and Stell G 1982 Phys. Rev. A 253302

[6] Lutsko J F 1996 Phys. Rev. Lett. 772225

[7] Grad H 1958 Encyclopedia of Physics ed S Flugge (New York: Springer)

[8] Ernst M H and Cohen E G D 1981 J. Stat. Phys. 25153

[9] Dufty J W 2001 Adv. Complex Syst. 4397

[10] Brey J J, García de Soria M I, Maynar P and Ruiz-Montero M J 2004 Phys. Rev. E 70011302

[11] Brey J J, Dufty J W, Kim C S and Santos A 1998 Phys. Rev. E 584638

[12] Dufty J W and Brey J J 2002 J. Stat. Phys. 109433

[13] Brey J J, Dufty J W and Ruiz-Montero M J 2003 Granular Gas Dynamics ed T Pöschel and N Brilliantov (New York: Springer) p 227

[14] Dufty J W and Brey J J 2003 Phys. Rev. E 68030302

[15] Dufty J W and Brey J J 2004 Modelling and Numerics of Kinetic Dissipative Systems ed L Pareschi, G Russo and G Toscani (New York: Nova Science) at press

[16] Lun C K K, Savage S B, Jeffrey D J and Chepurnity N 1984 J. Fluid Mech. 140223

[17] Jenkins J T and Richman 1985 Arch. Ration. Mech. Anal. 87355

Jenkins J T and Richman 1986 Phys. Fluids 283485

[18] Goldshtein A and Shapiro M 1995 J. Fluid Mech. 28275

[19] Brey J, Ruiz-Montero M J and Moreno F 2001 Phys. Rev. E 63061305

[20] Helal K, Biben T and Hansen J P 1997 Physica A 240361

[21] Ramírez R and Soto R 2003 Physica 32273

[22] Clement E and Rajchenbach J 1991 Europhys. Lett. 16133

[23] Wildman R D, Huntley J M and Parker D J 2001 Phys. Rev. E 63061311

[24] Blair D and Kudrolli A 2003 Phys. Rev. E 67041301

[25] Huang C, Yang X, Candela D, Mair R W and Walsworth R L 2004 Phys. Rev. E 69041302

[26] Brey J J and Ruiz-Montero M J 2004 Europhys. Lett. 66805

[27] Sela N and Goldhirsch I 1998 J. Fluid Mech. 36141

[28] Brey J J and Cubero D 2001 Granular Gases ed T Pöschel and S Luding (New York: Springer) p 59

[29] Lutsko J F 2001 Phys. Rev. E 63061211

[30] Brey J J, Ruiz-Montero M J and Moreno F 2004 Phys. Rev. E 69051303

[31] Bird G 1994 Molecular Gas Dynamics and the Direct Simulation of Gas Flows (Oxford: Clarendon)

[32] Brey J J and Ruiz-Montero M J 2004 Phys. Rev. E 70051301

[33] Goldhirsch I and Zanetti G 1993 Phys. Rev. Lett. 701619

[34] Brey J J, Ruiz-Montero M J and Cubero D 1996 Phys. Rev. E 543664

[35] van Noije T P C, Ernst M, Brito R and Orza J A C 1997 Phys. Rev. Lett. 79411

[36] Brey J J, Moreno F and Ruiz-Montero M J 1998 Phys. Fluids 102965

[37] Soto R, Piasecki J and Mareschal M 2001 Phys. Rev. E 64031306

[38] Pöschel T, Brilliantov N V and Schwager T 2002 Int. J. Mod. Phys. C 131263

[39] McLennan J A 1989 Introduction to Nonequilibrium Statistical Mechanics (Englewood Cliffs, NJ: Prentice-Hall) 